\title{
Adaptive Power Saving Receiver for DVB-H Exploiting Adaptive Modulation and Coding
}

\author{
Tallal Osama ELSHABRAWY, Sherif Hassan Abdel WAHED \\ Faculty of Information Engineering \& Technology, German University, Cairo, Egypt \\ Email:Tallal.El-Shabrawy@guc.edu.eg,Sherif.Mohamed@student.guc.edu.eg \\ Received November 12, 2009; revised December 11, 2009; accepted January 18, 2010
}

\begin{abstract}
Broadcasting live digital TV to a small battery-powered handheld device is very challenging. One of the most promising technologies to provide such services is DVB-H (Digital Video Broadcasting over Handheld). Power consumption has always been one of the most crucial challenges for handheld devices. In this paper, a novel Adaptive Modulation and Coding (AMC) framework is proposed for DVB-H systems to address the challenging problem of power consumption. The proposed power saving AMC framework operates by rearranging the transmitted frames in a pre-defined pattern. The adaptive receiver selects the appropriate modulation technique and/or code rate, one that achieves a target Bit Error Rate (BER), and then could be switched off and/or powered down resulting in significant potential for saving of reception and processing powers. Simulation of the DVB-H system under the proposed framework proved that the proposed power saving AMC framework is capable of achieving power saving up to $71.875 \%$ in COST207 Typical Urban 6-paths (TU6) channel. Furthermore, numerical analysis for the power saving potential and BER performance of the proposed framework is performed for both flat Rayleigh channel and multipath TU6 channel.
\end{abstract}

Keywords: Broadcast, DVB-H, Power Consumption, Power Saving, Adaptive Modulation and Coding, AMC, RCPC Codes.

\section{Introduction}

Multimedia services demand has grown rapidly in the previous decade. As a result, the Digital Video Broadcasting (DVB) project was founded in 1993 by the European Telecommunications Standards Institute (ETSI) with the goal of standardizing digital television services. In order to meet market demands, the DVB project released several standards regulating digital video broadcasting via satellite (DVB-S), cable (DVB-C), and terrestrial television (DVB-T) [1].

Recently along with the wireless era, there emerged a growing demand for multimedia services over handheld devices (defined as small and lightweight battery-powered devices). Accordingly, the ETSI ratified the standard for digital video broadcasting for handheld devices (DVB-H) in November 2004 [2], which is an amendment of the DVB-T standard [3] for handheld devices. DVB-H specifications were defined to achieve IP (Internet Protocol) data broadcasting to handheld devices. One of the most promising features of DVB-H is its synergy with interactive cellular platforms such as GPRS/UMTS $[4,6]$, allowing for IP-based interactive broadcasting "on the move". Since its release, DVB-H technology has been employed worldwide by a number of mobile operators in order to add new multimedia services such as Mobile TV [7]. In March 2008, DVB-H was officially endorsed by European Union as the preferred technology for terrestrial mobile broadcasting [8].

The DVB-H is a superset of DVB-T with additional three main features to meet the specific requirements of battery-powered handheld devices:

-MPE-FEC: Multi-Protocol Encapsulation - Forward Error Correction (MPE-FEC) employs a powerful channel coding on top of the DVB-T channel coding. Intensive testing of DVB-H showed that MPE-FEC reduces Signal to Noise Ratio (SNR) requirements up to $8 \mathrm{~dB}$ [9].

-4K OFDM Mode: A new 4K Orthogonal Frequency Division Multiplexing (OFDM) mode is introduced in order to facilitate network planning. The new $4 \mathrm{~K}$ mode offers a compromise between the good Doppler performance of the $2 \mathrm{~K}$ mode and the good suitability for large Single Frequency Networks of the 8K mode.

-Time-Slicing: Time-Slicing operates by transmitting data in bursts corresponding to a single service rather than continuously multiplexed with other services. No 
data is transmitted for a service between two consecutive bursts. Time-slicing is capable of reducing the receiver average power consumption up to $90 \%$ by switching off the receiver between consecutive bursts $[9,10]$.

Handheld devices are usually small and lightweight to facilitate ease of mobility. Hence, batteries are restricted to be small and lightweight as well resulting in a low power battery. Therefore, battery life time is a crucial limitation for handheld devices and should be used efficiently. DVB-H addressed this problem by introducing time slicing which is able to reduce the receiver average power consumption up to $90 \%$. Since then, a new field of research emerged looking for techniques to reduce the power consumption furthermore.

Power saving techniques for DVB-H could be categorized into cooperative techniques and compression techniques. The power saving potential in DVB-H networks exploiting cooperation among handheld devices is investigated in [11]. It is shown using numerical results that power saving of over $50 \%$ can be achieved by cooperative networks of three handheld devices in fully cooperating mode. The power saving potential due to employing progressive video codecs is studied in [12]. It is proposed to use progressive video codecs to provide a trade-off between video quality and receiver power consumption. JPEG2000 proved to be the best compression algorithm as it allows the receiver to control power consumption depending on quality level.

Adaptive Modulation and Coding (AMC), first introduced in the late 1970s [13], is one of the promising techniques to reduce power consumption. In [14], it is shown that AMC could result in power savings (at the transmitter) up to $40 \%$ over fading channels. However, AMC is impractical for broadcast systems as it is unfeasible to transmit at different code rates and modulation schemes to each user. To adopt the AMC concept in broadcast systems, the transmitter must adapt to the user (i.e. receiver) experiencing the worst channel. This constitutes a penalty to other users experiencing good channels.

In this paper, a novel power saving AMC framework is proposed for DVB-H systems. The proposed framework features three power saving schemes: Adaptive Coding (AC), Adaptive Modulation (AM), or Adaptive Modulation and Coding (AMC), in order to reduce the receiver power consumption significantly. The novelty arises from the fact that the proposed framework is initiated from receiver in contrast to traditional AMC schemes that rely on the existence of a feedback channel (from receiver to transmitter). Such feedback channels are unfeasible in broadcast systems. It is shown that the proposed framework is capable of achieving power savings up to $71.875 \%$. The proposed power saving AMC framework operates by rearranging the transmitted frames in a pre-defined pattern. The adaptive receiver selects the appropriate modulation technique and/or code rate, one that achieves a target Bit Error Rate (BER), and then could be switched off and/or powered down resulting in significant potential for saving of reception and processing powers.

A power saving approach similar to the proposed power saving AC scheme is introduced in [15]. This approach saves power by leaving out some FEC columns in the MPE-FEC frame once the receiver has received all the error-free data packets instead of always receiving the full frame. The maximum power saving potential for this approach is limited to $25 \%$. The power saving AC scheme proposed in this paper operates rather over the physical layer and could reap the benefits from switching off and/or powering down circuitry components from the receiver frontend in saving both reception and processing powers where receiving a bit stream usually consumes much more power than processing it.

The organization of this paper is as follows. In Section 2 , the DVB-H system is briefly illustrated. In Section 3, the novel power saving AMC framework is proposed. In Section 4, theoretical performance of the proposed framework is analyzed numerically. In Section 5, simulation results are given to illustrate the power saving potential of the proposed framework. Finally, a conclusion is given in Section 6.

\section{DVB-H System}

\subsection{System Overview}

DVB-H transmits IP-based services. The IP datagrams are typically produced via MPEG-4/AVC coding of video/audio signals. The IP datagrams are encapsulated by the IP encapsulator. The output is then modulated by a Coded OFDM (COFDM) modulator with $2 \mathrm{~K}, 4 \mathrm{~K}$, or $8 \mathrm{~K}$ carriers. The modulation scheme used could be QPSK, 16-QAM, or 64-QAM. The transmitted signal is organized into frames. Each OFDM frame consists of 68 OFDM symbols. Four frames constitute one super frame.

\subsection{Channel Coding}

The DVB-H system employs powerful concatenated channel coding consisting of a variable rate punctured Convolutional code as the inner code and a Reed-Solomon (RS) code as the outer code. This combination provides great performance due to the optimum performance

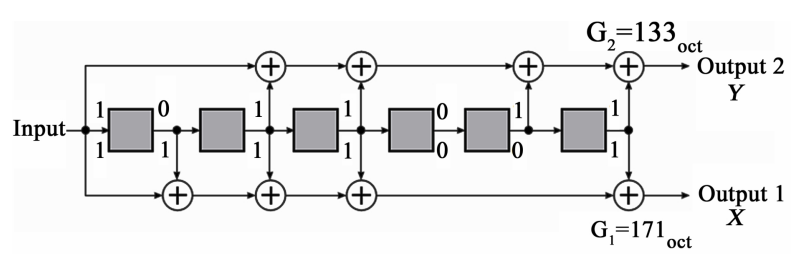

Figure 1. Schematic diagram of DVB-H conv. encoder. 
of Viterbi decoder and ability of RS code to correct burst errors produced by Viterbi decoder.

The DVB-H standard employs a $(204,188)$ shortened RS code that corrects up to 8 erroneous bytes in a received word. In addition, it employs a variable rate punctured convolutional code based on a powerful $1 / 2$ mother code compiled by Odenwalder [16]. A schematic diagram of the convolutional encoder is shown in Figure 1. The code rates supported by DVB-H are 1/2, 2/3, 3/4, 5/6, and $7 / 8$. The puncturing patterns for the different code rates are given in Table 1.

Table 1. Puncturing patterns for different code rates [17].

\begin{tabular}{|c|c|}
\hline Code Rate & Puncturing Pattern \\
\hline \multirow{2}{*}{$1 / 2$} & $\mathrm{X}: 1$ \\
\hline & Y: 1 \\
\hline \multirow{2}{*}{$2 / 3$} & $\mathrm{X}: 10$ \\
\hline & $Y: 11$ \\
\hline \multirow{2}{*}{$3 / 4$} & $X: 101$ \\
\hline & $Y: 110$ \\
\hline \multirow{2}{*}{$5 / 6$} & $X: 10101$ \\
\hline & $Y: 11010$ \\
\hline \multirow{2}{*}{$7 / 8$} & $X: 1000101$ \\
\hline & Y: 1111110010 \\
\hline
\end{tabular}

1 and 0 denote transmitting and puncturing of coded bits, respectively.

Table 2. Power saving of DVB-H'S code vs RCPC code.

\begin{tabular}{lcccc}
\hline Code Rate & $2 / 3$ & $3 / 4$ & $5 / 6$ & $7 / 8$ \\
\hline DVB-H Power Saving (\%) & 1.9 & 5.71 & 17.14 & 42.86 \\
RCPC Power Saving (\%) & 25 & 33.33 & 40 & 42.86 \\
\hline
\end{tabular}

Power saving of different code rates was calculated compared to worstcase of receiving the whole transmit frame (i.e. code rate 1/2).

Table 3. Puncturing patterns for Hagenauer RCPC codes [18] $(M=6$ and $P=8)$.

\begin{tabular}{|c|c|c|c|}
\hline Code Rate & Puncturing & Pattern & \multirow{11}{*}{ 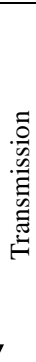 } \\
\hline \multirow{2}{*}{$8 / 9$} & $\mathrm{X}: 1111$ & 0111 & \\
\hline & Y: 1000 & 1000 & \\
\hline \multirow{2}{*}{$4 / 5$} & $\mathrm{X}: 1111$ & 1111 & \\
\hline & Y: 1000 & 1000 & \\
\hline \multirow{2}{*}{$2 / 3$} & $\mathrm{X}: 1111$ & 1111 & \\
\hline & Y: 1010 & 1010 & \\
\hline \multirow{2}{*}{$4 / 7$} & $\mathrm{X}: 1111$ & 1111 & \\
\hline & $Y: 1110$ & 1110 & \\
\hline \multirow{2}{*}{$1 / 2$} & X: 1111 & 1111 & \\
\hline & $\mathrm{Y}: 1111$ & 1111 & \\
\hline
\end{tabular}

Table 4. Puncturing patterms for proposed AC scheme employing Hagenauer RCPC codes $(M=6$ and $P=8)$.

\begin{tabular}{cccc}
\hline Code Rate & $\begin{array}{c}\text { Puncturing } \\
\text { Pattern }\end{array}$ & Puncturing Pattern \\
\hline \multirow{2}{*}{$8 / 9$} & $Q_{1}$ & $\mathrm{X}: 1111$ & 0111 \\
& & $\mathrm{Y}: 1000$ & 1000 \\
$4 / 5$ & $Q_{2}$ & $\mathrm{X}: 0000$ & 1000 \\
& & $\mathrm{Y}: 0000$ & 0000 \\
$2 / 3$ & $Q_{3}$ & $\mathrm{X}: 0000$ & 0000 \\
& & $\mathrm{Y}: 0010$ & 0010 \\
$4 / 7$ & $Q_{4}$ & $\mathrm{X}: 0000$ & 0000 \\
& & $\mathrm{Y}: 0100$ & 0100 \\
$1 / 2$ & $Q_{5}$ & $\mathrm{X}: 0000$ & 0000 \\
& & $\mathrm{Y}: 0001$ & 0001 \\
\hline
\end{tabular}

\section{Proposed Power Saving AMC Framework}

In this section, a novel power saving AMC framework is proposed for DVB-H systems. The three power saving schemes: adaptive coding, adaptive modulation, or adaptive modulation and coding, supported by the AMC framework are proposed in Subsections 3.1, 3.2, and 3.3, respectively. Hardware aspects of the proposed framework are discussed in Subsection 3.4.

\subsection{Novel Power Saving AC Scheme}

\subsubsection{Novel Power Saving AC Scheme Framework}

Since for broadcast systems the transmit power is much less crucial than handheld receiver power, therefore transmission using the strongest code (i.e. lowest code rate) is proposed in the power saving $\mathrm{AC}$ scheme introduced in this paper. The transmitted frame is arranged in such a way that higher code rates (i.e. weaker codes) are transmitted first and then incremental bits (constituting stronger codes) are transmitted afterwards. The incremental bits are chosen according to pre-defined puncturing patterns. This gives the receiver the flexibility to receive few more bits in order to upgrade the code to a stronger one, in case higher rate codes are not sufficiently powerful to meet the target BER. The transmitted frame size defines how often the code rate is allowed to change (i.e. adaptation rate). The adaptation rate is a very important design parameter that depends on time variation of the channel (i.e. Doppler frequency).

On the other end, the adaptive power saving receiver selects the appropriate code rate, one that achieves a target BER, according to certain thresholds and then could be switched off and/or powered down resulting in significant potential for saving of receiving and processing power. The adaptive receiver is incremental in nature. It works by initially receiving the weakest coded bits, and then using the received SNR a decision is made whether the receiver should receive the bits of the next stronger code.

\subsubsection{DVB-H Convolutional Code}

Employing DVB-H standard punctured convolutional code in the proposed scheme would face the following crucial limitation. Close inspection of the puncturing patterns given in Table 1 reveals that not all bits of high rate codes are used by lower rate codes. Therefore, by arranging bits of the transmit frame in accordance to the proposed scheme, the adaptive receiver would be occasionally forced to receive more bits than required for the decoding of stronger codes. This introduces losses in the power saving potential compared to the case of using ideal RCPC codes as shown in Table 2.

The power saving of the proposed AC scheme when RCPC code of rate $R_{i}$ is employed at the receiver could be simply calculated as follows, 


$$
P_{i}=\left(1-\frac{R_{W C}}{R_{i}}\right) \times 100
$$

where $R_{W C}$ denotes the worst case scenario code rate. The power saving of the proposed AC scheme when DVB-H convolutional code is employed was calculated numerically using puncturing patterns summarized in Table 1 .

\subsubsection{RCPC Codes}

In order to get over the crucial limitation of DVB-H's convolutional code, employment of RCPC codes is proposed. RCPC codes are variable rate punctured convolutional codes with rate-compatibility restriction on puncturing patterns. The rate-compatibility restriction ensures that all code bits of high rates code are used by the lower rate codes. RCPC codes were first introduced by Hagenauer in [18]. Hagenauer produced families of RCPC codes with rates between $8 / 9$ and $1 / 4$.

Hagenauer RCPC code with memory $M=6$ and puncturing period $P=8$, that is based on the same DVB-H standard $1 / 2$ mother code, is employed in the proposed AC scheme. The puncturing patterns of employed code rates are summarized in Table 3 where the bits in bold are the ones required to be transmitted by the proposed scheme for each code rate.

For example, the proposed power saving AC scheme employing Hagenauer RCPC code is illustrated in Figure 2. This scheme assumes portable reception (i.e. $v=$ $3 \mathrm{~km} / \mathrm{hr}$, thus channel is quasi-stationary over the period of one super frame). Hence, an adaptation rate of super frame is adopted. Each transmitted super frame is arranged such that higher code rates are transmitted first followed by incremental bits in order to upgrade the code. The incremental bits are chosen according to the pre-defined puncturing patterns summarized in Table 4. The adaptive receiver must rearrange the received bits according to the puncturing patterns given in Table 3 before the Viterbi decoder. Assume in Figure 2 that the receiver decides that the $4 / 5$ code is sufficient to meet the target BER. Then, the receiver will receive 170 instead of 272 OFDM symbols. This translates into a power saving of $37.5 \%$ compared to the worst case of receiving the whole super frame. The power saving of the proposed AC scheme employing Hagenauer RCPC code is summarized in Table 5. It is worth noting that generating RCPC code with the same code rates of DVB-H standard convolutional code would require a puncturing period of 210 which would make finding the best puncturing pattern for each code rate very tedious.

\subsection{Novel Power Saving AM Scheme}

\subsubsection{Novel Power Saving AM Scheme Framework}

In order to adopt the AM concept in broadcast systems, users experiencing good SNR conditions should be allocated a high modulation technique such as 16-QAM, while users experiencing bad SNR conditions should be allocated a more robust modulation technique such as QPSK. However, transmitting two different modulation techniques is unfeasible. Instead, it is proposed to combine both constellations in a hierarchical fashion where lower modulation symbols are embedded inside higher modulation ones. For instance, the constellation diagram of an AM scheme supporting QPSK and 16-QAM is depicted in Figure 3 where the two left most bits of each 16-QAM symbol could be treated as a QPSK symbol embedded inside the 16-QAM symbol. In this manner, only the two right most bits would not be retrieved by receivers employing QPSK. Hence, retransmission using QPSK modulation of those non-embedded bits is required.

Table 5. Power saving of proposed AC scheme employing Hagenauer RCPC code.

\begin{tabular}{lcccc}
\hline Code Rate & $2 / 3$ & $3 / 4$ & $5 / 6$ & $7 / 8$ \\
\hline Power Saving $(\%)$ & 12.5 & 25 & 37.5 & 43.75 \\
\hline
\end{tabular}

Power saving of different codes was calculated using (1).

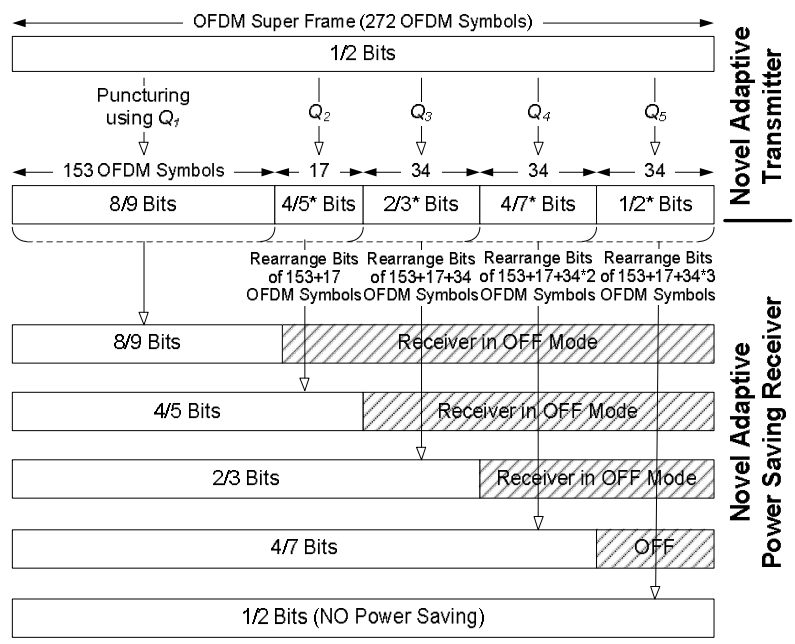

Figure 2. Schematic of the proposed AC scheme (adaptation rate: super frame).

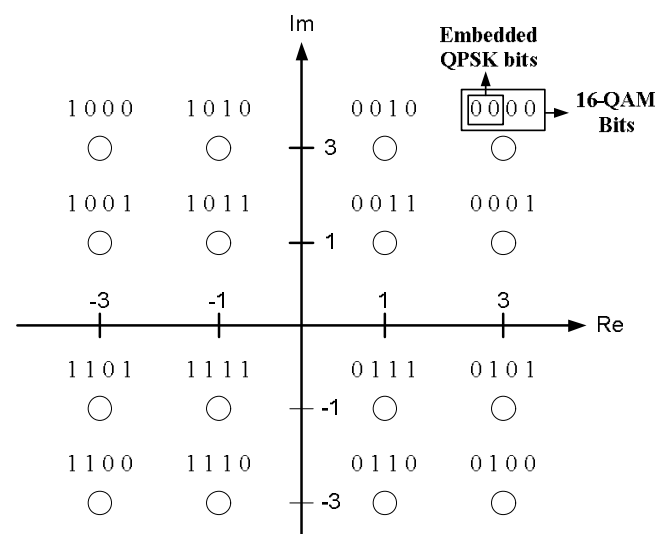

Figure 3. QPSK symbols embedded inside 16-QAM symbols. 
Accordingly in the proposed power saving AM scheme, the transmitted frame constituting a block of convolutionally coded bits is subdivided into $L$ clusters. In order to provide users (i.e. receivers) having good SNR conditions the highest power saving potential, the first cluster consists of all coded bits which are then modulated using the highest modulation technique (i.e. modulation technique having the largest constellation size). As a result, users having good SNR conditions are capable of reliably receiving the highest modulation symbols and then could switch off and/or power down their receiver frontend resulting in significant power saving potential. For users experiencing bad SNR conditions (where the highest modulation technique is insufficient to meet the target BER), the second cluster of the transmit frame consists of the non-embedded coded bits (i.e. coded bits that will be lost when employing lower modulation techniques such as QPSK) which are then modulated using a lower modulation technique. As a result, users having bad SNR conditions are capable of receiving the first two clusters and then could switch off and/or power down their receiver frontend resulting in significant power saving potential. The remaining clusters are arranged in a similar fashion to accommodate coded bits that are not embedded in previous clusters (i.e. are not embedded in higher modulation symbols).

The transmitted frame size defines how often the modulation technique is allowed to change (i.e. adaptation rate). The adaptation rate is a very important design parameter that depends on time variation of the channel (i.e. Doppler frequency).

On the other end, the adaptive power saving receiver selects the appropriate modulation technique, one that achieves a target BER, according to certain thresholds and then could be switched off and/or powered down

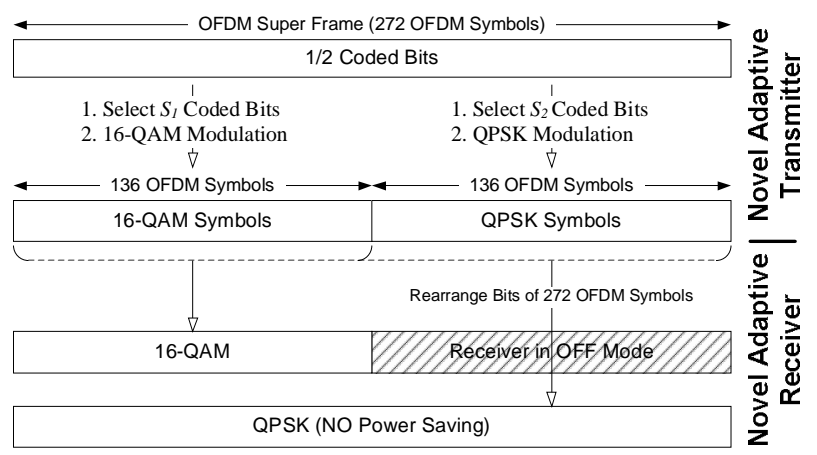

Figure 4. Schematic of proposed AM scheme (adaptation rate: super frame).

Table 6. Selection patterns employed by the proposed AM scheme.

\begin{tabular}{ccc}
\hline Modulation & Selection Pattern & \multicolumn{1}{c}{ Selected Bits } \\
\hline 16-QAM & $S_{l}$ & $1,2,3,4,5,6, \ldots, K$ \\
QPSK & $S_{2}$ & $1,2,5,6,9,10 \ldots, K-3, K-2$ \\
\hline
\end{tabular}

resulting in significant potential for saving of receiving and processing powers. The adaptive receiver is incremental in nature. It works by initially receiving the highest modulation symbols, and then using the received SNR a decision is made whether the receiver should receive the symbols of the next lower modulation technique.

\subsubsection{Employment of AM Scheme in DVB-H}

For example, the proposed power saving AM scheme employing two modulation techniques (QPSK and 16-QAM) is depicted in Figure 4. This scheme assumes portable reception (i.e. $v=3 \mathrm{~km} / \mathrm{hr}$, thus channel is quasistationary over the period of one super frame). Hence, an adaptation rate of super frame is adopted. Furthermore, $1 / 2$ rate convolutional code is employed in order to maintain reliable performance in bad SNR conditions. Each transmitted super frame is arranged such that higher modulation symbols (in this example 16-QAM symbols) are transmitted first followed by lower modulation symbols (in this example QPSK symbols). The selected bits are chosen according to the pre-defined selection patterns summarized in Table 6. Assume the receiver decides that the 16-QAM is sufficient to meet the target BER. Then, the receiver will receive 136 instead of 272 OFDM symbols. This translates into a power saving of $50 \%$ compared to the worst case of receiving the whole super frame.

\subsubsection{Power Saving Potential}

The power saving of the proposed AM scheme when modulation technique $M_{i}$ is employed at the receiver could be simply calculated as follows,

$$
P_{i}=\left(1-\frac{\log _{2}\left(M_{W C}\right)}{\log _{2}\left(M_{i}\right)}\right) \times 100
$$

where $M_{W C}$ denotes the worst case scenario modulation technique. The power saving for the three modulation techniques supported by DVB-H is given in Table 7.

\subsection{Novel Power Saving AMC Scheme}

\subsubsection{Novel Power Saving AMC Scheme Framework}

In the proposed AMC scheme, the transmitted frame is arranged in such a way that higher modulation symbols are transmitted first followed by lower modulation symbols comprising symbols that were not embedded in higher modulation. Those non-embedded symbols must be retransmitted as receivers employing lower modulation technique will not be able to retrieve them (similar to proposed AM scheme, see Figure 3). Each $M_{i}$-QAM ( $i$ $=1,2, \ldots, L)$ transmitted symbols are arranged such that higher code rates (i.e. weaker codes) are transmitted first followed by incremental bits (constituting stronger codes). The incremental bits are chosen according to predefined puncturing patterns. The transmitted frame size defines how often the modulation technique and code 
rate are allowed to change (i.e. adaptation rate). The adaptation rate is a very important design parameter that depends on time variation of the channel (i.e. Doppler frequency).

\subsubsection{Employment of AMC Scheme in DVB-H}

For example, the proposed AMC scheme employing two modulation techniques (QPSK and 16-QAM) and five code rates is depicted in Figure 5. This scheme assumes portable reception (i.e. $v=3 \mathrm{~km} / \mathrm{hr}$, thus channel is quasistationary over the period of one super frame). Hence, an adaptation rate of super frame is adopted. The transmitted bits are first rearranged using the five puncturing patterns summarized in Table 4. Then, the bits are further divided into two groups according to the selection patterns summarized in Table 6. Finally, the first and second group of bits $\left(S_{1}\right.$ and $\left.S_{2}\right)$ are modulated using 16-QAM and QPSK modulation techniques, respectively.

The adaptive receiver selects the appropriate modulation and coding configuration, one that achieves a target BER, according to pre-defined SNR thresholds and then could switch off and/or reduce power from any possible circuitry in the frontend resulting in significant potential for saving of reception and processing powers. The receiver is incremental in nature. It works by initially receiving bits of the highest modulation technique and weakest code (i.e. 16-QAM, 8/9), and then using the received SNR a decision is made whether the receiver should receive symbols of the next stronger code. Assume the receiver decides that 16-QAM and 2/3 code are sufficient to meet the target BER. Then, the receiver will receive and process 102 instead of 272 OFDM symbols. This translates into a power saving of $62.5 \%$.

\subsubsection{Power Saving Potential}

The power saving of the proposed AMC scheme when modulation technique $M_{i}$ and code rate $R_{j}$ are employed at the receiver could be simply calculated as follows,

$$
P_{i, j}=\frac{\log _{2}\left(M_{W C}\right)}{\log _{2}\left(M_{B C}\right)}\left(\frac{\log _{2}\left(M_{i}\right)}{\log _{2}\left(M_{W C}\right)}-\frac{R_{W C}}{R_{j}}\right) \times 100
$$

Table 7. Power saving of proposed AM scheme.

\begin{tabular}{lccc}
\hline Modulation & QPSK & 16-QAM & 64-QAM \\
\hline Power Saving (\%) & - & 50 & 66.67 \\
\hline
\end{tabular}

Power saving of different modulation techniques calculated compared to worst-case scenario of receiving the whole transmit frame (i.e. QPSK).

Table 8. Power saving (\%) of proposed AMC scheme.

\begin{tabular}{cccccc}
\hline \multirow{2}{*}{ Modulation } & \multicolumn{5}{c}{ Code Rate } \\
\cline { 2 - 6 } & $1 / 2$ & $4 / 7$ & $2 / 3$ & $4 / 5$ & $8 / 9$ \\
\hline 16-QAM & 50 & 56.25 & 62.5 & 68.75 & 71.875 \\
QPSK & - & 6.25 & 12.50 & 18.75 & 21.875 \\
\hline
\end{tabular}

Power saving of different AMC configurations was calculated compared to worst-case scenario of receiving whole transmit frame (i.e. QPSK, 1/2). where $M_{W C}$ and $M_{B C}$ denote the worst and best case modulation technique, respectively. $R_{W C}$ depicts the worst case code rate. Equation (3) is valid only for a scheme supporting two modulation techniques. Power saving of the proposed AMC scheme is given in Table 8.

\subsection{Hardware Aspects}

It is emphasized that the proposed AMC framework operates at the physical layer leading to power saving potential in the Radio Frequency (RF) frontend (a main source of battery power drainage in DVB-H devices). By switching off the receiver, this do not necessarily mean the receiver is going to sleep mode. As in conventional TDMA systems, the receiver during inactivity periods has the ability to switch off and/or reduce power from any possible circuitry in the frontend [19]. In the proposed AMC framework, power reduction could be achieved by using a DC-to-DC converter [20] to reduce supply voltage during receiver inactivity periods. Typical supported switching rate in DC-to-DC converters is 6 $\mathrm{MHz}$ (i.e. $0.16 \mu \mathrm{s}$ ), for example; Texas Instruments TPS62601. This high switching rate achieves the adaptation rates employed by the proposed framework.

\section{Numerical Analysis}

In this section, the theoretical performance of the proposed power saving AMC framework is analyzed numerically. For simplicity and without loss of generality,

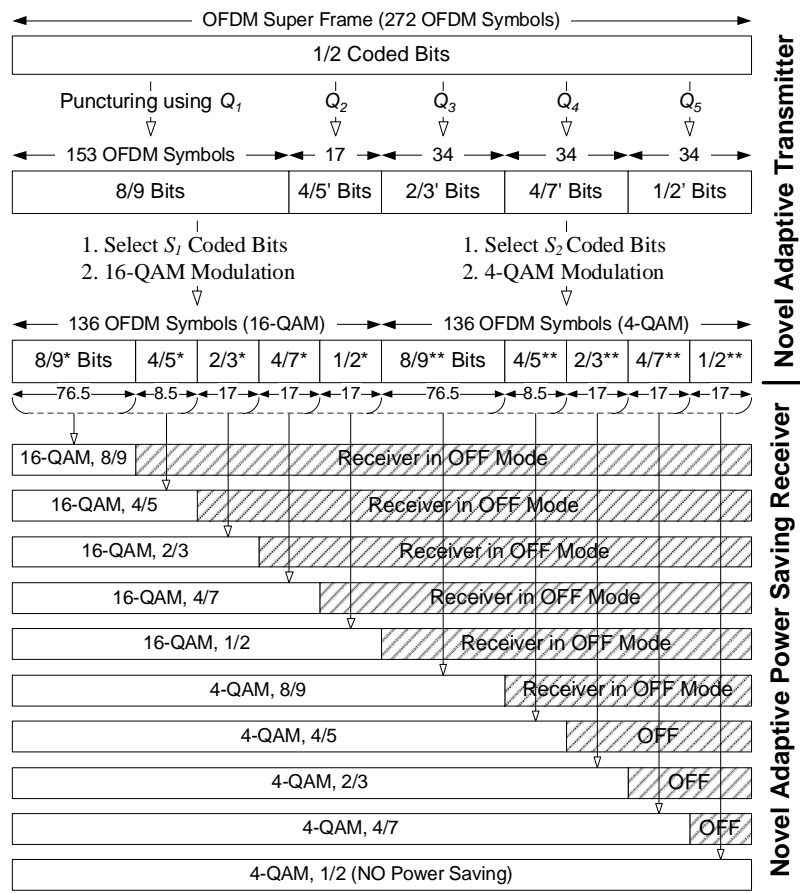

Figure 5. Schematic of proposed AMC scheme (adaptation rate: super frame). 


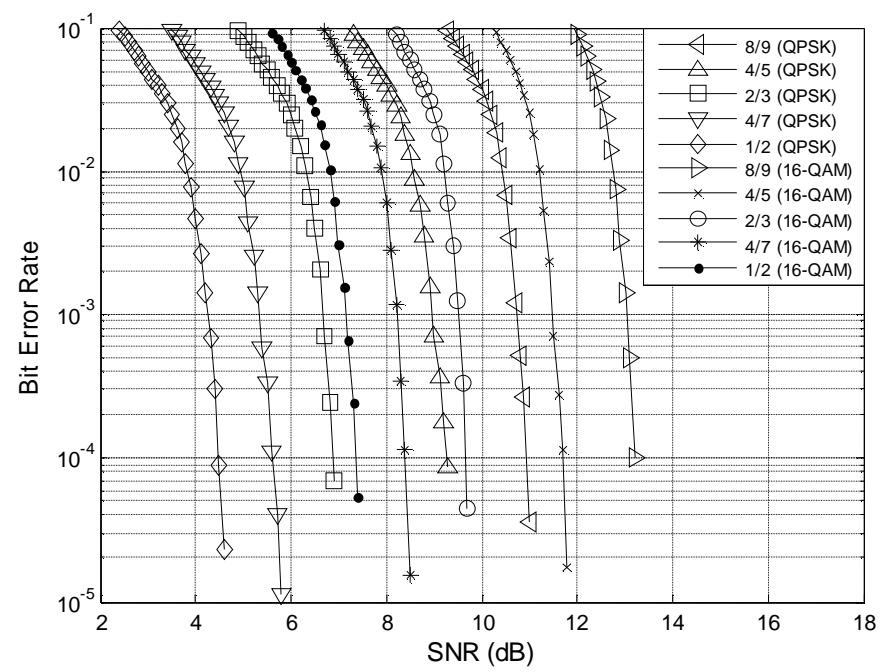

Figure 6. BER performance of fixed-rate AMC scheme in AWGN channel.

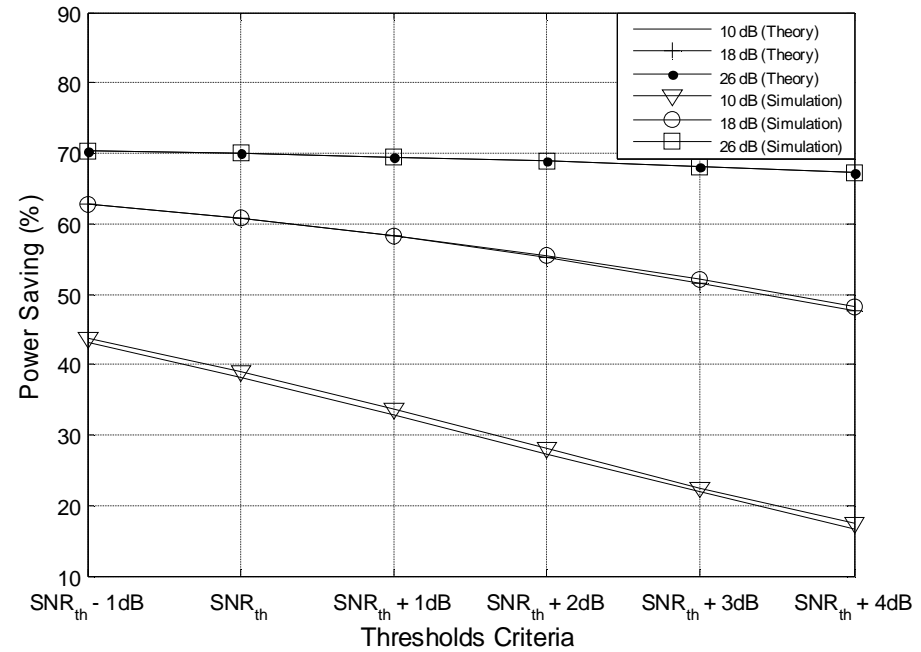

Figure 7. Power saving of proposed AMC scheme in flat Rayleigh channel.

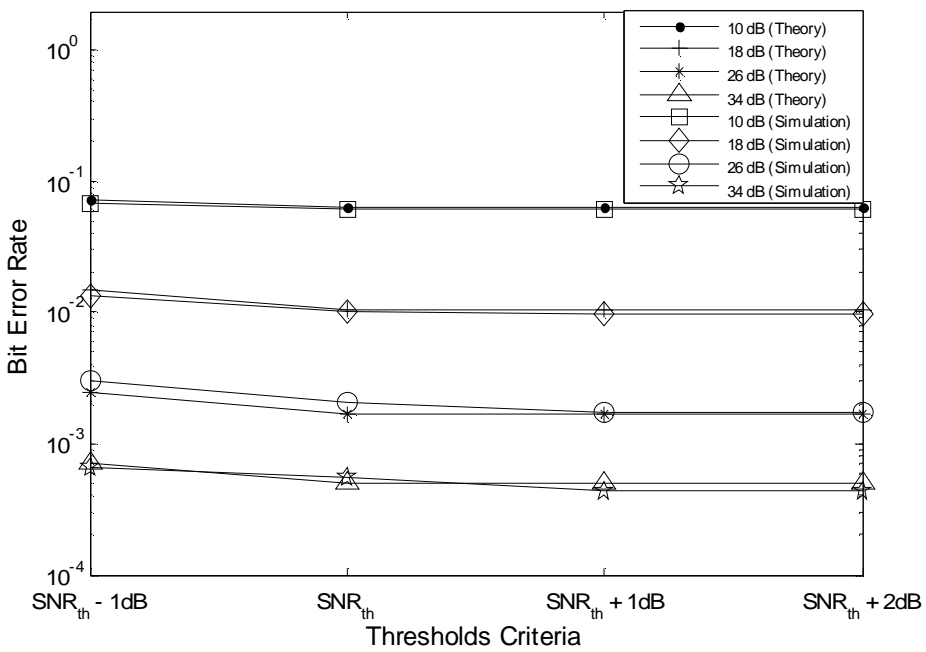

Figure 8. BER performance of proposed AMC scheme in flat Rayleigh channel. 
perfect Channel State Information (CSI) is assumed at the receiver. The numerical analysis is performed for the generic case of an AMC scheme. The numerical analysis of AC and AM schemes could be regarded as special cases. The numerical analysis is performed in two environments: flat Rayleigh channel and COST207 Typical Urban 6-paths (TU6) channel [21]. The power delay profile of the TU6 channel is given in Section 5.1.

\subsection{Flat Fading Channel}

The power saving is given by,

$$
P_{\text {Saving }}=\sum_{i=1}^{L} \sum_{j=1}^{N} P_{i, j} p_{i, j}
$$

where $P_{i, j}$ denotes the power saving due to employing modulation technique $M_{i}$ and code $R_{j}$ at the adaptive receiver and is given by (3). $L$ and $N$ are the number of modulation techniques and code rates supported by the proposed AMC scheme. $p_{i, j}$ depicts the probability of selecting modulation technique $M_{i}$ and code $R_{j}$, and is given as,

$$
p_{i, j}=\int_{x_{i, j}^{(L)}}^{x_{i, j}^{(U)}} f_{X}(x) d x
$$

where $x_{i, j}^{(L)}$ and $x_{i, j}^{(U)}$ denote the lower and upper SNR thresholds of using modulation technique $M_{i}$ and code $R_{j}$, respectively. $f_{X}(x)$ is the Probability Density Function (PDF) of the received SNR. Since a Rayleigh channel is considered, the received SNR follows an exponential distribution. Hence, $f_{X}(x)$ is defined as,

$$
f_{X}(x)=\frac{1}{\lambda} e^{-\frac{x}{\lambda}} \quad x \geq 0
$$

where $\lambda$ depicts the average channel SNR.

The BER in flat Rayleigh channel is given by,

$$
B E R=\sum_{i=1}^{L} \sum_{j=1}^{N} \int_{x_{i, j}^{(L)}}^{x_{i, j}^{(U)}} B E R_{i, j}(x) f_{X}(x) d x
$$

where $B E R_{i, j}(x)$ denotes the BER due to employing modulation technique $M_{i}$ and code $R_{j}$ at the adaptive receiver while having SNR $x$. It is obtained from the Monte Carlo simulations of DVB-H system in AWGN given in Figure 6.

The appropriate modulation and code are selected by comparing the SNR to a set of predefined thresholds that guarantee a target BER. A target BER of $10^{-3}$ is chosen. The $10^{-3}$ SNR thresholds (depicted $\mathrm{SNR}_{\mathrm{th}}$ in Figs.) could be obtained easily from Figure 6. Such thresholds are incrementally increased and decreased by steps of $1 \mathrm{~dB}$ to provide a tradeoff between power saving and BER. The simulation results depicted in Figure 6 show that (16-QAM, 1/2) mode performs better than (QPSK, 4/5) and (QPSK, 8/9) modes while providing better power saving. As a result, those two modes were eliminated from the proposed AMC scheme.

Figures 7 and 8 illustrate the power saving and BER of the proposed scheme, respectively, where the modulation and code rate are allowed to change every convolutional block through-out the simulations (The DVB-H standard defines the convolutional $1 / 2$ coded block to be 3264 bits). Simulation results are in excellent agreement with theoretical results. The BER performance is limited by the deep fades of the Rayleigh channel. This explains the BER floor experienced in the low SNR region where the AMC scheme cannot guarantee target BER anymore (even with the aid of $1 / 2$ code). At SNR of $26 \mathrm{~dB}$, the AMC scheme is capable of achieving almost its full power saving potential of $71 \%$.

\subsection{Multipath TU6 Channel}

For simplicity and without loss of generality, COST207 TU6 channel having Doppler frequency of $40 \mathrm{~Hz}$ and an AMC scheme employing an adaptation rate of quarter frame (i.e. modulation and coding configuration is allowed to change every 17 OFDM symbols) are considered. Numerical analysis of the BER performance in TU6 multipath channel is very tedious due to the frequency-selective nature of the channel. To the best of the authors' knowledge, there is no upper bound on the BER performance of OFDM employing concatenated RS-convolutional codes in frequency-selective fading channels due to the complicated nature of this problem. However, an approximate method is adopted in order to perform numerical analysis of the BER performance in the frequency-selective TU6 channel. The approximate BER estimation method works by averaging the SNR in time (i.e., over the quarter frame interval) and frequency (i.e. across sub-carriers) referred to as mean quarter frame SNR. Then, the BER could be obtained from BER performance of DVB-H system employing proposed AMC scheme in $40 \mathrm{~Hz}$ TU6 channel (depicted in Figure 9). Hence, Equation (7) could be used to deduce the BER performance where $B E R_{i, j}(x)$ denotes the BER due to employing modulation technique $M_{j}$ and code rate $R_{i}$ at the adaptive receiver while having $x$ as the mean quarter frame SNR. $B E R_{i}(x)$ is obtained from Figure 9. For the distribution of the mean quarter frame SNR, $f_{X}(x)$ employed in Equations (5), (7) is approximated as a log-normal distribution where the PDF of the received mean quarter frame SNR is defined as,

$$
f_{X}(x)=\frac{1}{x \sigma \sqrt{2 \pi}} e^{-\frac{(\ln x-\mu)^{2}}{2 \sigma^{2}}} \quad x \geq 0
$$

where $\mu$ and $\sigma$ depict the average and standard deviation of the nature logarithm of $x$, the mean quarter frame SNR.

Based on simulation experiments carried on a $40 \mathrm{~Hz}$ 


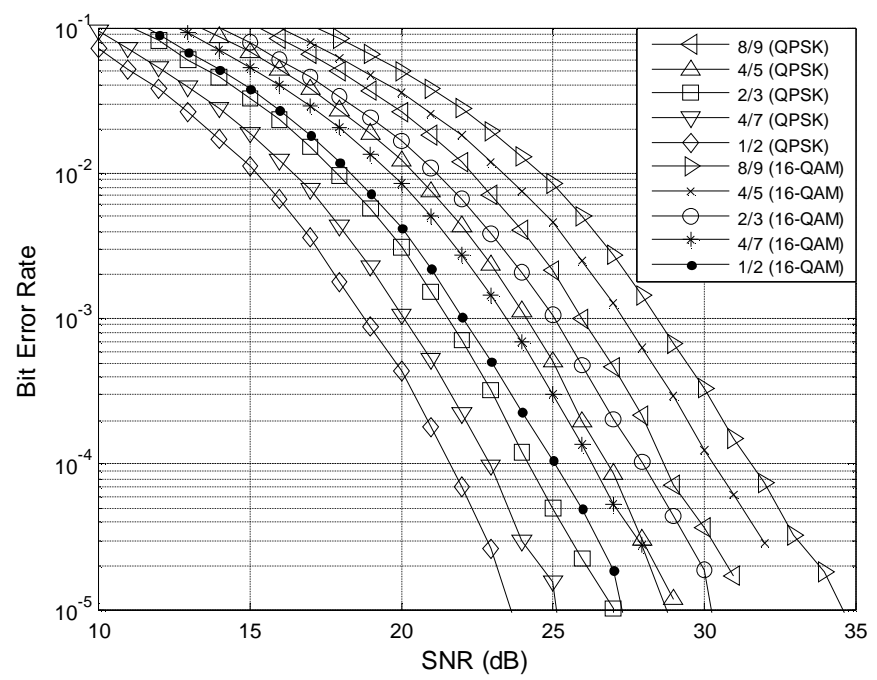

Figure 9. BER performance of fixed-rate AMC scheme in $40 \mathrm{~Hz}$ TU6 channel.

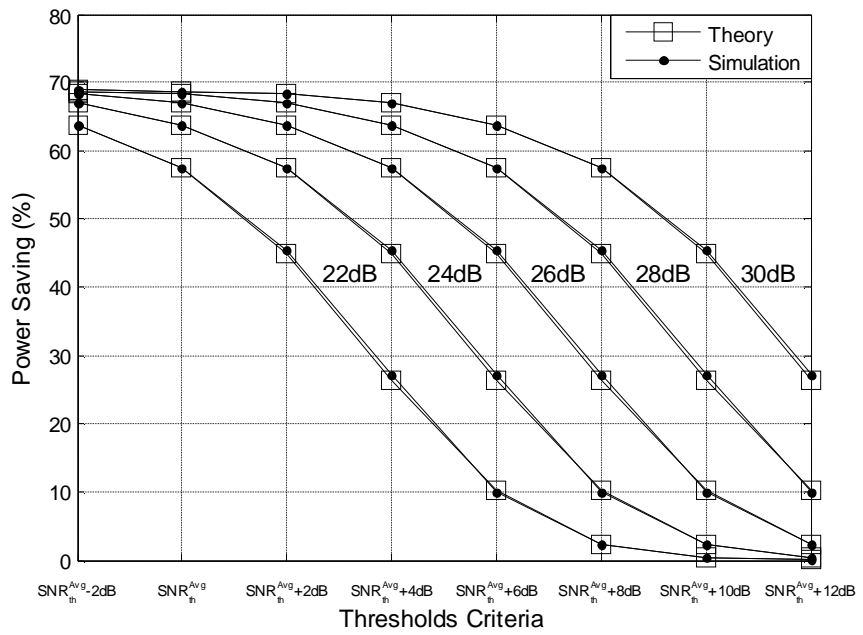

Figure 10. Power saving of proposed AMC scheme in $40 \mathrm{~Hz}$ TU6 channel.

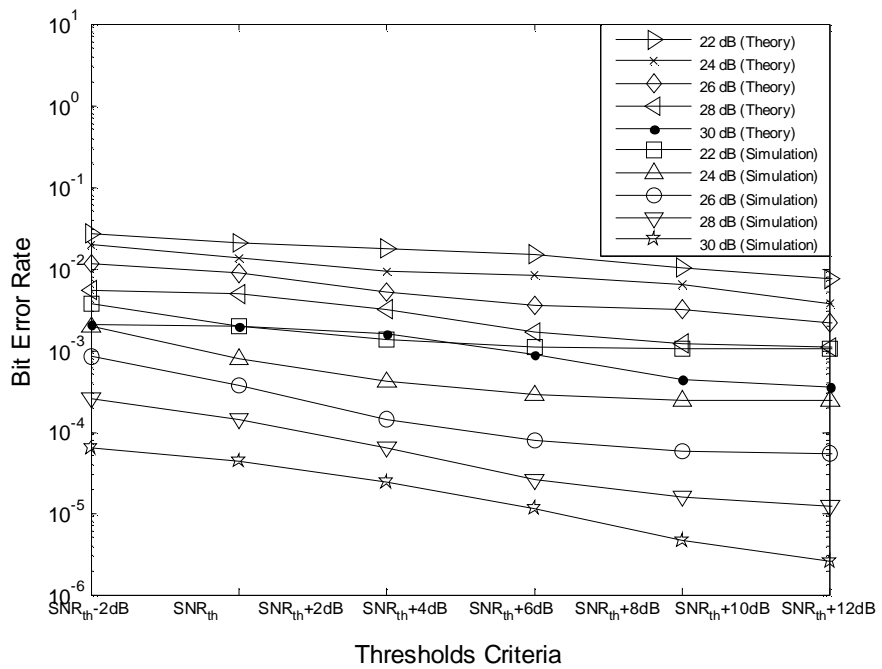

Figure 11. BER performance of proposed AMC scheme in $40 \mathrm{~Hz}$ TU6 channel. 
TU6 channel, the PDF of the received mean quarter frame SNR could fit a log-normal distribution with parameters:

- $\mu=4.046256349081641$

- $\sigma=0.529152417564057$

The appropriate modulation and coding configuration is selected by comparing the mean quarter frame SNR to a set of predefined SNR thresholds that guarantee a target BER. A target BER of $10^{-3}$ is chosen. The assignment of the $10^{-3}$ SNR thresholds is defined in the next section. These thresholds are incrementally increased and decreased by steps of $2 \mathrm{~dB}$ to provide a tradeoff between power saving and BER. Figures 10 and 11 illustrate the power saving and BER of the proposed AMC scheme, respectively. The simulation results of the power saving potential are in excellent agreement with theoretical ones. However, the simulation results of BER performance are better than theoretical ones. As a result, the theoretical BER results (obtained via proposed approximate method) could be regarded as an upper bound on the BER performance.

\section{Simulation Results}

In this section, the simulation results of DVB-H system employing the proposed power saving AMC framework are given and discussed comprehensively. Section 5.1 defines the channel model and adaptation rates used throughout the simulations. Section 5.2 summarizes the simulation parameters of the DVB-H system employing the proposed AMC framework. Simulation results illustrating power saving potential and BER performance of DVB-H system employing AC, AM, and AMC schemes are given in Sections 5.3, 5.4, and 5.5, respectively. Finally, the three power saving schemes are compared in Section 5.6.

Table 9. TU6 channel power delay profile.

\begin{tabular}{ccccccc}
\hline Tap Number & 1 & 2 & 3 & 4 & 5 & 6 \\
\hline Delay $(\mu \mathrm{s})$ & 0.0 & 0.2 & 0.5 & 1.6 & 2.3 & 5.0 \\
Power $(\mathrm{dB})$ & -3 & 0 & -2 & -6 & -8 & -10 \\
Doppler Spectrum & & \multicolumn{5}{c}{ Rayleigh } \\
\hline
\end{tabular}

Table 10. Reception model and velocity range for different Doppler Frequencies.

\begin{tabular}{ccc}
\hline Doppler Frequency & Receiver & Velocity Range \\
\hline $10 \mathrm{~Hz}$ & $\begin{array}{c}\text { Moderately Mo- } \\
\text { bile }\end{array}$ & $13.5-54 \mathrm{~km} / \mathrm{h}$ \\
$40 \mathrm{~Hz}$ & Severely Mobile & $54-216 \mathrm{~km} / \mathrm{h}$ \\
\hline
\end{tabular}

Table 11. SNR thresholds (dB) (adaptation rate: quarter frame, 40HZ TU6 channel).

\begin{tabular}{lcccc}
\hline Code Rate & $8 / 9$ & $4 / 5$ & $2 / 3$ & $4 / 7$ \\
\hline SNR Threshold & {$[18, \infty[$} & ] $16,18]$ & ] $13,16]$ & ] $11,13]$ \\
\hline
\end{tabular}

\subsection{Channel Model and Adaptation Rates}

The COST207 TU6 channel model [21] has proven to be a very good representative for typical mobile reception. The power delay profile of the TU6 channel is given in Table 9. In order to model various types of receivers, two Doppler frequencies were considered. The Doppler frequencies along with their corresponding velocity ranges are summarized in Table 10. The velocity range is calculated starting from carrier frequency $800 \mathrm{MHz}$ (upper part of Band V) to carrier frequency $200 \mathrm{MHz}$ (lower part of Band III).

Accordingly, the proposed power saving schemes were implemented employing three adaptation rates (to accommodate different Doppler frequencies and to provide a trade-off between performance and complexity). The three supported adaptation rates are: super frame, frame and quarter frame (where as the name implies, the modulation and/or coding configuration is allowed to change every super frame, frame, and quarter frame corresponding to 272,68 , and 17 OFDM symbols, respectively).

\subsection{Simulation Model}

For simulation experiments, a DVB-H system is employed assuming 2K OFDM mode with 1/4 guard interval. The DVB-H standard convolutional code is replaced by the Hagenauer RCPC code in order to exploit full power saving potential. $8 \mathrm{MHz}$ channel is considered. Perfect CSI is assumed at the receiver. Simulations are run for 500 OFDM super frames. MATLAB/SIMULINK [22] is the simulation tool used. For simulations of DVB-H employing the proposed AC scheme, an AC scheme that supports five code rates $(1 / 2,4 / 7,2 / 3,4 / 5$, and 8/9) is considered. Furthermore, QPSK is chosen as the modulation technique. For simulations of DVB-H employing the proposed AM scheme, an AM scheme that supports two modulation techniques (QPSK and 16-QAM) is considered. In addition, the convolutional code rate is fixed to $1 / 2$. For simulations of DVB-H employing the proposed AMC scheme, an AMC scheme that supports two modulation techniques (QPSK and 16-QAM) and five code rates $(1 / 2,4 / 7,2 / 3,4 / 5$, and $8 / 9)$ is considered.

\subsection{Power Saving AC Scheme}

For TU6 multipath channel, defining the SNR thresholds is challenging due to the frequency-selective nature of the channel. Hence, the mean SNR (averaged over time and frequency, i.e. across OFDM subcarriers) is adopted as the decision criteria. A target BER of $10^{-3}$ is defined. The $10^{-3}$ average thresholds (depicted $\mathrm{SNR}_{\text {th }}^{\mathrm{Avg}}$ in Figures.) were obtained by gathering statistics of appropriate code rates that guarantee the target BER averaged over 


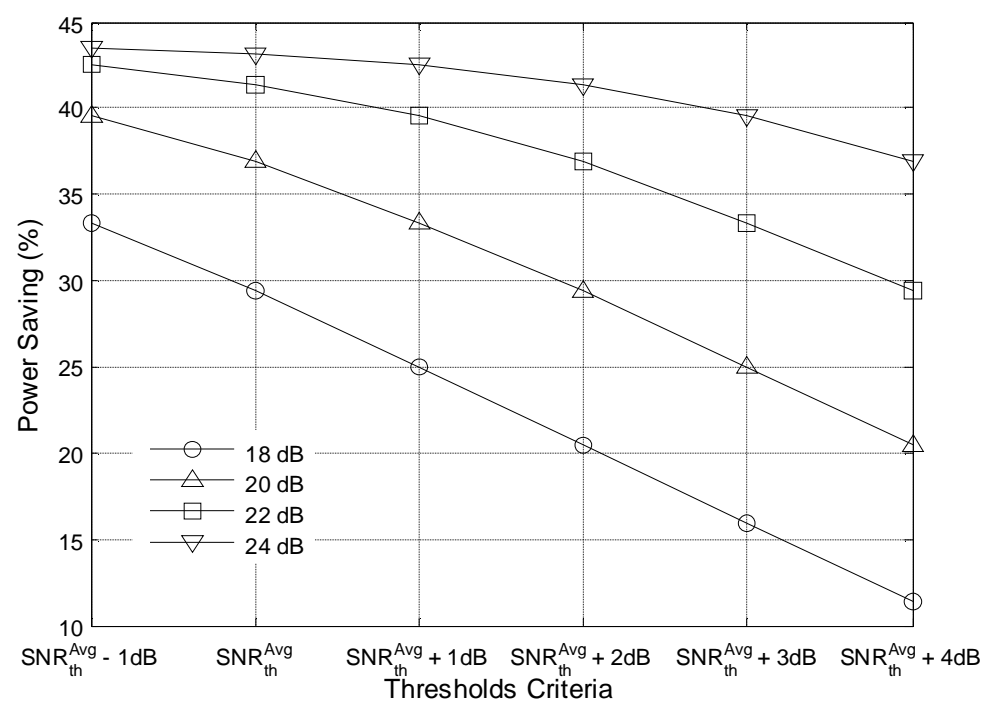

Figure 12. Power savings of proposed AC scheme in $40 \mathrm{~Hz}$ TU6 channel.

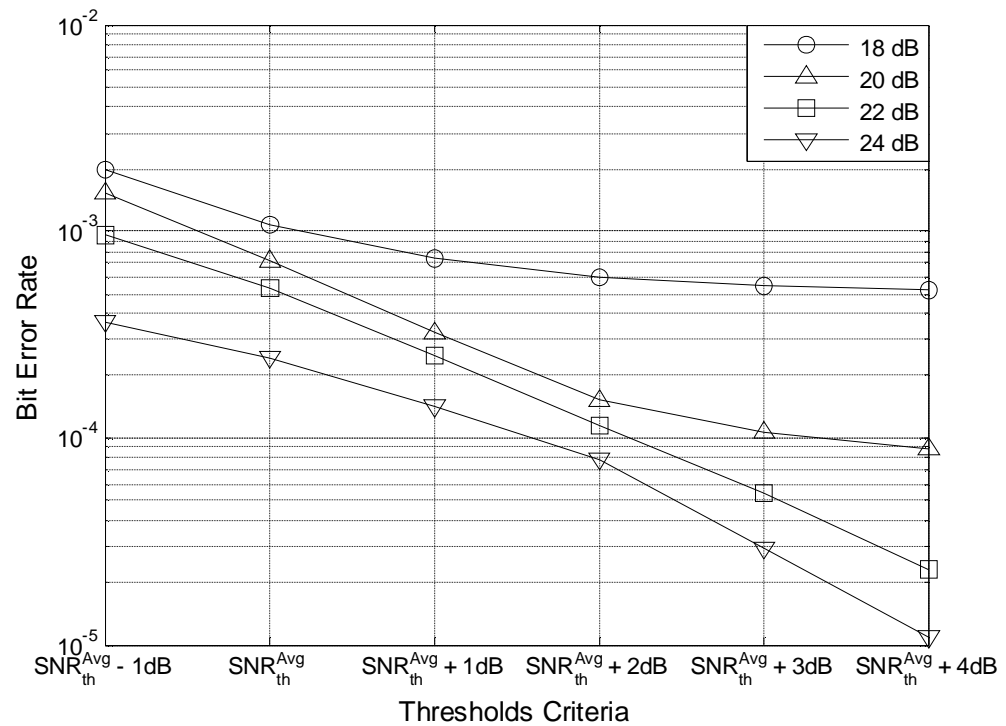

Figure 13. BER performance of proposed AC scheme in $40 \mathrm{~Hz}$ TU6 channel.

5000 super frames. For example, consider an AC scheme with an adaptation rate of quarter frame in a $40 \mathrm{~Hz}$ TU6 channel. The $10^{-3}$ thresholds are given in Table 11 . Power saving and BER of the AC scheme at different values of average channel SNR are depicted in Figures 12 and 13 , respectively.

Figures 12 and 13 demonstrate the tradeoff of increasing/decreasing the decision thresholds. Increasing the thresholds, improves the BER performance on the expense of power saving. Figures 12 and 13 could be combined as depicted in Figure 14. For a target BER, Figure 14 could be used to determine the corresponding power saving given the average channel SNR. Moreover, the appropriate thresholds to be employed by the receiver could be deduced. For example at average channel SNR of $18 \mathrm{~dB}$ power saving of approximately $29 \%$ could be achieved for a target BER of $10^{-3}$. At high average channel SNR, maximum power saving potential of $43.75 \%$ could be achieved.

The power savings versus average channel SNR that guarantee a target BER of $10^{-3}$ for different adaptation rates (super frame, frame, and quarter frame) and Doppler frequencies $(10 \mathrm{~Hz}$ and $40 \mathrm{~Hz})$ are summarized in Figure 15. As expected, increasing the adaptation rate helps the adaptive receiver increase the power saving potential particularly for fast fading channels. This is obvious for the $40 \mathrm{~Hz}$ channel where the AC scheme employing an adaptation rate of quarter frame saves more power than the one employing an adaptation rate of super frame. For instance at an average channel SNR of $18 \mathrm{~dB}$, the faster $\mathrm{AC}$ scheme saves $6 \%$ more power. In the $10 \mathrm{~Hz}$ channel, increasing the adaptation rate saves 


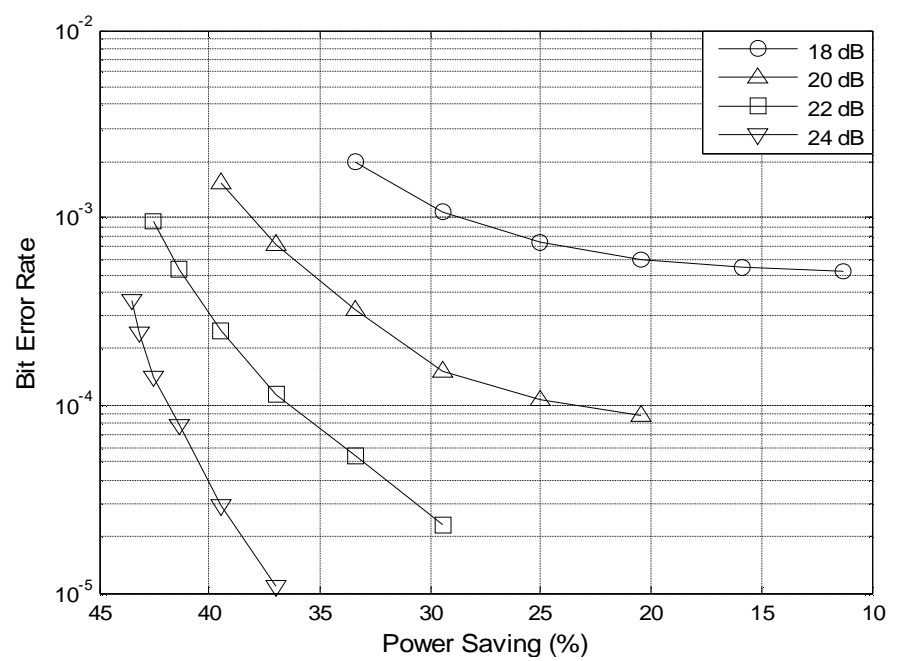

Figure 14. BER vs. power saving of proposed AC scheme in $40 \mathrm{~Hz}$ TU6 channel.

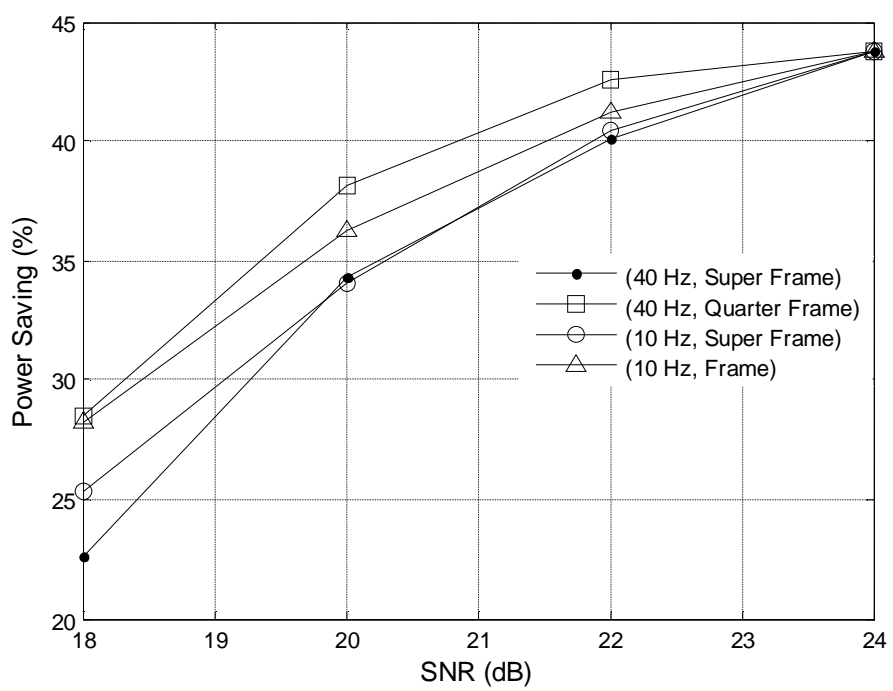

Figure 15. Power saving vs. SNR of proposed AC scheme for different adaptation rates and Doppler frequencies in TU6 channel (Target BER $=\mathbf{1 0}^{-3}$ ).

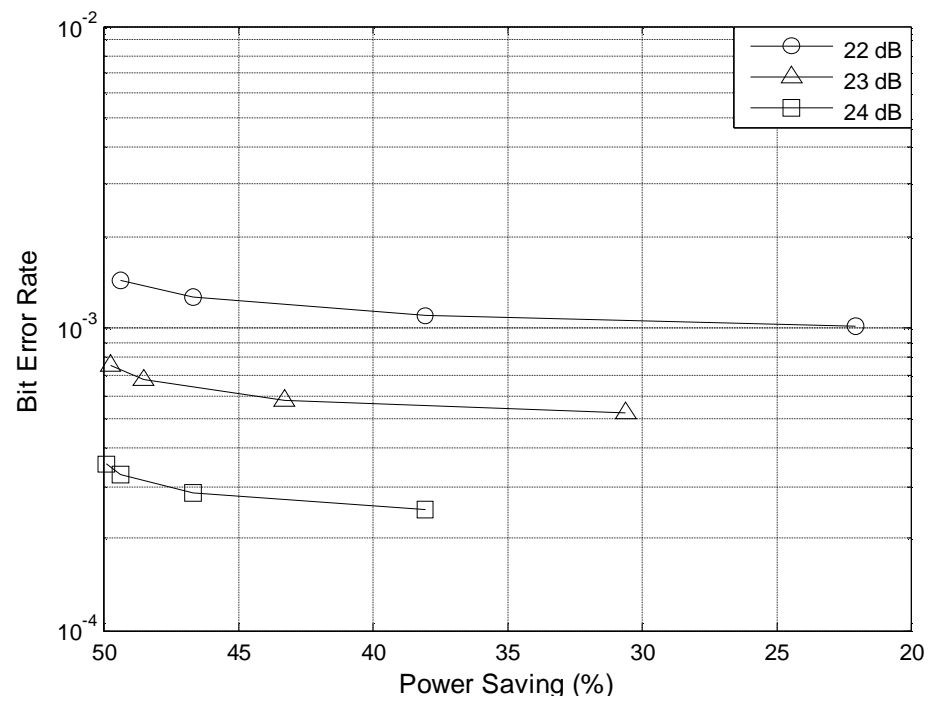

Figure 16. BER vs. power saving of proposed AM scheme in $40 \mathrm{~Hz}$ TU6 channel. 
$3 \%$ more power at $18 \mathrm{~dB}$. At high average channel SNR, all schemes achieve maximum power saving potential of $43.75 \%$.

\subsection{Power Saving AM Scheme}

Consider an AM scheme with an adaptation rate of quarter frame in a $40 \mathrm{~Hz}$ TU6 channel. The $10^{-3}$ SNR thresholds could be defined in a similar manner to AC scheme. The power saving versus the BER performance is shown in Figure 16. For a target BER, Figure 16 could be used to determine the corresponding power saving given the average channel SNR. Moreover, the appropriate thresholds to be employed by the receiver could be deduced. For instance, at average channel SNR of $22 \mathrm{~dB}$ power saving of $22 \%$ could be achieved. At high average channel SNR, maximum power saving potential of $50 \%$ could be achieved.

\subsection{Power Saving AMC Scheme}

Consider an AMC scheme with an adaptation rate of quarter frame in a $40 \mathrm{~Hz}$ TU6 channel. The $10^{-3} \mathrm{SNR}$ thresholds could be obtained in a similar manner to AC scheme. The power saving versus the BER performance is shown in Figure 17. For a target BER, Figure 17 could be used to determine the corresponding power saving given average channel SNR. Moreover, the appropriate thresholds to be employed by the adaptive receiver could be deduced. For instance, at average channel SNR of 22 $\mathrm{dB}$ power saving of $30 \%$ could be achieved. At high average channel SNR, maximum power saving potential of $71.875 \%$ could be achieved.

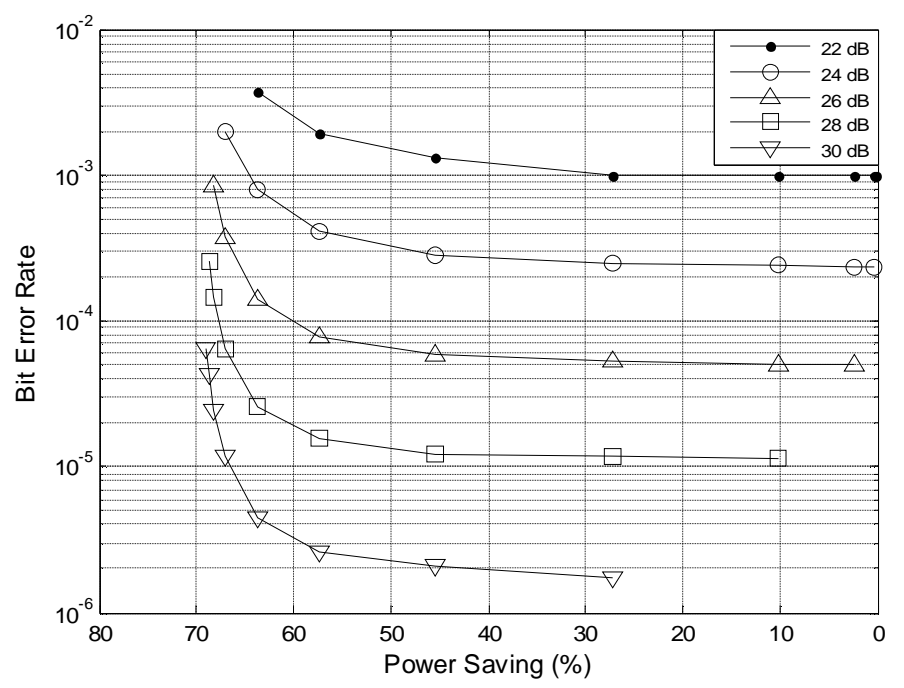

Figure 17. BER vs. power saving of proposed AMC in $40 \mathrm{~Hz}$ TU6 channel.

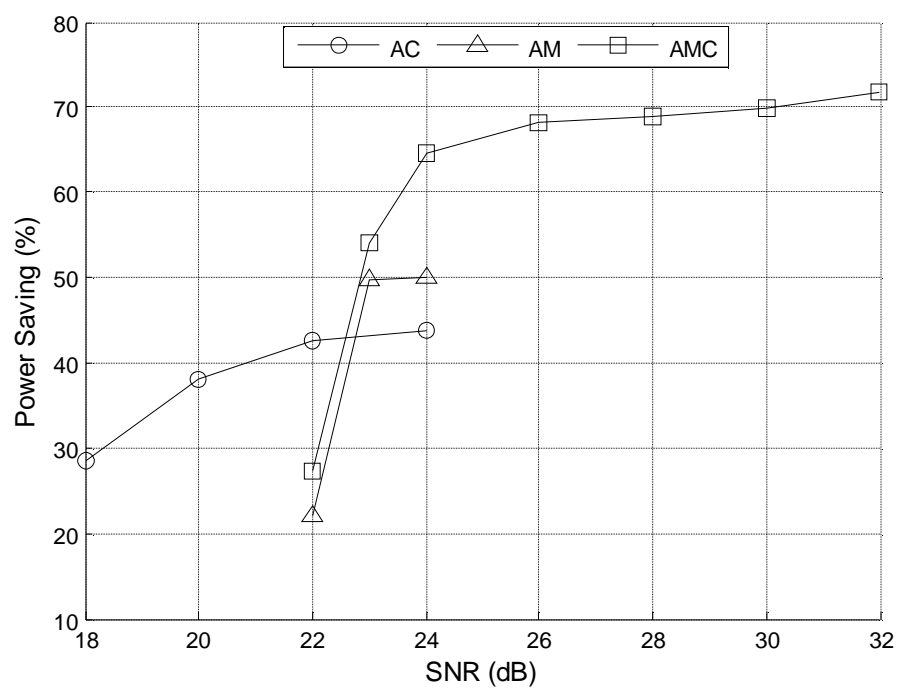

Figure 18. Power saving vs. average channel SNR of the proposed three power saving schemes in 40 Hz TU6 channel (target BER $=10^{-3}$. 


\subsection{Comparison of Three Power Saving Schemes}

The proposed three power saving schemes are compared in terms of power saving potential in Figure 18 The proposed AC scheme provides significant power saving (28.5-43.75\%) for a wide range of SNRs (18-24 dB). On the other hand, the proposed AM scheme provides more power saving $(50 \%)$ compared to the AC scheme but for a narrower range of SNRs $(23-24 \mathrm{~dB})$. This narrow range of SNRs is due to the limited power saving modes (only two modulation techniques, opposed to the five code rates supported by the $\mathrm{AC}$ scheme). Note that in the low SNR region (18-22 dB) the AC scheme provide higher power saving potential than AM scheme where at very low SNRs $(18-20 \mathrm{~dB})$ the AM scheme fails to provide any power saving for the target BER of $10^{-3}$.

For high average channel SNR (above approximately $22.5 \mathrm{~dB}$ ), the proposed AMC scheme is clearly the better choice. It provides significant power saving (42-71.87$5 \%$ ) for a very wide range of SNRs (22.5-32 dB). The proposed AMC scheme is unable to provide any power saving (for the target BER of $10^{-3}$ ) in the low SNR region $(18-20 \mathrm{~dB})$ due to the fact that the QPSK modulation technique is actually embedded inside the 16-QAM constellation (see Figure 3) and hence making it much less robust than the normal non-embedded QPSK modulation technique employed by the AC scheme. This also justifies why the AC scheme outperforms the AM scheme in the very low SNR region.

\section{Conclusions}

In this paper, a novel power saving AMC framework was proposed for DVB-H systems to address the challenging problem of power consumption. The proposed framework features three schemes: adaptive coding, adaptive modulation, and adaptive modulation/coding in order to reduce the receiver power consumption. The proposed AMC framework is initiated from receiver in contrast to traditional AMC schemes that rely on the existence of a feedback channel. It operates by rearranging transmitted frames in a pre-defined pattern. The adaptive receiver selects the appropriate modulation and/or coding configuration, one that achieves a target BER, and then could be switched off and/or powered down resulting in significant potential for saving of reception and processing powers. It was shown that the proposed power saving AMC framework is capable of achieving remarkable power saving (up to $71.875 \%$ ) in TU6 channel environment.

Furthermore, numerical analysis for the power saving potential and BER performance of the proposed AMC framework is performed where theoretical ones were shown to be in excellent agreement with simulation results for the case of Rayleigh channel. For TU6 channel, the numerical results could serve as an upper bound on the BER performance of the proposed scheme.

In addition, the effect of adaptation rate on power saving was studied for different Doppler frequencies. As expected, increasing the adaptation rate helps the adaptive receiver increase the power saving potential particularly for fast fading channels. This is obvious for the $40 \mathrm{~Hz}$ TU6 channel where the proposed AC scheme employing an adaptation rate of quarter frame saves $6 \%$ more power than the one employing an adaptation rate of super frame.

Finally, the three schemes supported by the proposed framework (AC, AM, and AMC) were compared in terms of power saving potential. It was shown that for environments characterized by high average channel SNR (greater than $22.5 \mathrm{~dB}$ ), the AMC scheme would be the best scheme where $42-71.875 \%$ power saving was achieved for the target BER of $10^{-3}$. On the other hand, for environments characterized by low SNR (less than $22.5 \mathrm{~dB}$ ), the AC scheme would yield the highest power saving potential $(28.5-42 \%)$.

\section{References}

[1] U. Reimers, "DVB - The family of international standards for digital video broadcasting," in Proceedings of IEEE, Vol. 94, No. 1, January 2006.

[2] ETSI EN 302 304: "Digital Video Broadcasting (DVB); transmission system for handheld terminals (DVB-H)," European Telecommunication Standard, November 2004.

[3] ETSI EN 300 744: "Digital Video Broadcasting (DVB); framing structure, channel coding and modulation for digital terrestrial television (DVB-T)", European Telecommunication Standard, January 2004.

[4] C. Rauch, W. Kellerer, and P. Sties, "Hybrid mobile interactive services combining DVB-T and GPRS," in Proceedings of European Personal Mobile Communication Conference, Vienna, Austria, February 2001.

[5] E. Stare and S. Lindgren, "Hybrid broadcast-telecom systems for spectrum efficient mobile broadband internet access," in Nordic Radio Symposium, Sweden, 2001.

[6] G. Gardikis, G. Kormentzas, G. Xilouris, H. Koumaras, and A. Kourtis, "Broadband data access over hybrid DVB-T networks," in Proceedings of 3rd Conference on Heterogeneous Network, Ilkley, UK, July 2005.

[7] A. Kumar, "Mobile TV: DVB-H, DMB, 3G systems and rich media applications," Focal Press, 2007.

[8] J. Gozalvez, "The european union backs the DVB-H standard [mobile radio]," IEEE Vehicular Technology Magazine, Vol. 3, pp. 3-12, June 2008.

[9] ETSI TR 102 401: "Digital Video Broadcasting (DVB); transmission system for handheld terminals (DVB-H)," Validation Task Force Report, May 2005.

[10] G. Gardikis, H. Kokkinis, and G. Kormentzas, "Evaluation of the DVB-H data link layer," in Proceedings of European Wireless, Paris, France, April 2007.

[11] Q. Zhang, F. H. P. Fitzek, and M. Katz, "Cooperative 
power saving strategies for IP-services supported over DVB-H networks," in Proceedings of IEEE Wireless Communication and Network Conference, March 2007.

[12] E. Belyaev, T. Koski, J. Paavola, A. Turlikov, and A. Ukhanva, "Adaptive power saving on the receiver side in digital video broadcasting systems based on progressive video codec," The 11th International Symposium on Wireless Personal Multimedia Communication, 2008.

[13] J. F. Hayes, “Adaptive feedback communications," IEEE Transactions on Communication Technology, Vol. COM16, pp. 29-34, February 1978.

[14] A. Goldsmith and S. G. Chua, "Adaptive coded modulation for fading channels," IEEE Transactions on Communication, Vol. 46, pp. 595-602, May 1998.

[15] E. D. Balaguer, F. H. P. Fitzek, and O. Olsen, "Performance evaluation of power saving strategies for DVB-H services using adaptive MPE-FEC decoding," The 16th Annual IEEE International Symposium on Personal Indoor and Mobile Radio Communication, Berlin, Germany, September 2005.

[16] J. Odenwalder, "Optimal decoding of convolutional codes,
"Ph.D. dissertation, Department of Systems Sciences, School of Engineering and Applied Sciences, University of California, Los Angeles, 1970.

[17] Y. Yasuda, K. Kashiki, and Y. Hirata, "High-rate punctured convolutional codes for soft decision viterbi decoding," IEEE Transactions on Communication, Vol. COM-32, pp. 315-319, March 1984.

[18] J. Hagenauer, "Rate-compatible punctured convolutional Codes (RCPC Codes) and their applications," IEEE Transactions on Communication, Vol. 36, pp. 389-400, April 1988.

[19] T. S. Rappaport. Wireless communications: Principles \& practice," Prentice Hall, New Jersey, 1996.

[20] F. L. Luo, and H. Ye, Essential DC/DC Converters. CRC Press, 2006.

[21] COST207, "Digital land mobile communications," Commission of the European Communities, Directorate General Communications, Information Industries and Innovation, 1989, pp. 135-147.

[22] A. Gilat, "MATLAB: An introduction with applications," $3^{\text {rd }}$ Edition, John Wiley and Sons, 2008. 